\title{
THE RELATIVE DEGREE ENHANCEMENT PROBLEM FOR MIMO NONLINEAR SYSTEMS ${ }^{1}$
}

D. A. Schoenwald

Instrumentation and Controls Division

Oak Ridge National Laboratory

P. O. Box 2009

Oak Ridge, TN 37831-8066
Ü. Özgüner

Department of Electrical Engineering

The Ohio State University

2015 Neil Avenue

Columbus, $\mathrm{OH} 43210-1272$

To be presented at the

IFAC Symposium on Nonlinear Control Systems Design

Tahoe City, California

June 25-28, 1995

\begin{abstract}
"The submitted manuscript has been authored by a contractor of the U.S. Government under contract No. DE-AC05-84OR21400.

Accordingly, the U.S. Government retains a nonexclusive, royalty-free license to publish or reproduce the published form of this contribution, or allow others to do so, for U.S. Government purposes."
\end{abstract}

\footnotetext{
${ }^{1}$ Research sponsored by the Laboratory Directed Research Program of Oak Ridge National Laboratory, managed for the Department of Energy by Martin Marietta Energy Systems, Inc., under Contract No. DE-AC05-84OR21400.
}

$\int_{2}$ 


\section{DISCLAIMER}

This report was prepared as an account of work sponsored by an agency of the United States Government. Neither the United States Government nor any agency thereof, nor any of their employees, make any warranty, express or implied, or assumes any legal liability or responsibility for the accuracy, completeness, or usefulness of any information, apparatus, product, or process disclosed, or represents that its use would not infringe privately owned rights. Reference herein to any specific commercial product, process, or service by trade name, trademark, manufacturer, or otherwise does not necessarily constitute or imply its endorsement, recommendation, or favoring by the United States Government or any agency thereof. The views and opinions of authors expressed herein do not necessarily state or reflect those of the United States Government or any agency thereof. 


\section{DISCLAIMER}

Portions of this document may be illegible in electronic image products. Images are produced from the best available original document. 


\title{
THE RELATIVE DEGREE ENHANCEMENT PROBLEM FOR MIMO NONLINEAR SYSTEMS*
}

\author{
D. A. SCHOENWALD**and Ü. ÖZGÜNER ${ }^{* * *}$ \\ *Research sponsored in part by the U. S. Department of Energy under Contract DE-ACO5- \\ 84 OR21400 with Martin Marietta Energy Systems, Inc. \\ **Instrumentation and Controls Division, Oak Ridge National Laboratory, P. O. Box 2009, Oak \\ Ridge, TN 37831-8066 U.S.A. E-Mail: schoenwaldda@ornl.gav \\ ***Department of Electrical Engineering, The Ohio State University, $2015 \mathrm{Neil}$ Avenue, Columbus, \\ OH 43210-1272 U.S.A. E-Mail: umit@ee.eng.ohio-state.edu
}

\begin{abstract}
We present a result for linearizing a nonlinear MIMO system by employing partial feedback - feedback at all but one input-output channel such that the SISO feedback linearization problem is solvable at the remaining input-output channel. The partial feedback effectively enhances the relative degree at the open input-output channel provided the feedback functions are chosen to satisfy relative degree requirements. The method is useful for nonlinear systems that are not feedback linearizable in a MIMO sense. Several examples are presented to show how these feedback functions can be computed. This strategy can be combined with decentralized observers for a completely decentralized feedback linearization result for at least one input-output channel.
\end{abstract}

Key Words. Feedback linearization; decentralized control; MIMO; partial differential equations; nonlinear systems

\section{PROBLEM STATEMENT}

We start by looking at $n$th order nonlinear systems with $m$ inputs and $m$ outputs

$$
\begin{aligned}
\dot{x} & =f(x)+\sum_{i=1}^{m} g_{i}(x) u_{i} \\
y_{i} & =h_{i}(x), \quad i=1, \ldots, m
\end{aligned}
$$

where it is assumed that $f(x)$ and $g_{i}(x)$ are smooth vector fields, and $h_{i}(x)$ are smooth functions defined on an open set of $R^{n}$. We seek control laws of the form $u_{i}=K_{i}\left(y_{i}, v_{i}\right), i=1, \ldots, m$ which will render the system (1) linear with a nonlinear change of coordinates. The $v_{i}$ are external inputs available to control the linearized system. In general, the output feedback functions $K_{i}$ will be nonlinear, and we assume they are smooth. It is also assumed in this paper that the individual input-output pairs $\left(u_{i}, y_{i}\right)$ are SISO, though this is easily generalized to multivariable input-output channels. An additional assumption is that the control vector fields $g_{i}(x)$ form a complete set about a given point $x^{0}$.

The MIMO exact linearization problem has been solved in Cheng et al. (1988), and a key concept in the proof of this result is that of a vector relative degree. For a SISO nonlinear system the relative degree is the number of times one must differentiate the output until the input appears explicitly.
For a MIMO nonlinear system the relative degree is a vector $\left\{r_{1}, \ldots, r_{m}\right\}$ wherein each $r_{i}$ is the number of times one has to differentiate the $i$ th output to have at least one of the $m$ inputs appear explicitly. The conditions for the existence of this relative degree, defined about some point $x^{0}$, are stated in Isidori (1989) and are not repeated here. The important point is that the state space exact linearization problem for (1) is solvable (about $x^{0}$ ) if and only if there exists some vector relative degree $\left\{r_{1}, \ldots, r_{m}\right\}$ at $x^{0}$ and $r_{1}+r_{2}+\cdots+r_{m}=n$.

If this relative degree condition is not satisfied and one has freedom to choose new output functions (say $y_{i}=\lambda_{i}(x)$ ) then there exist necessary and sufficient conditions to find these $\lambda_{i}(x)$ such that the relative degree condition is satisfied (see Isidori (1989) for details). If none of these conditions are satisfied then the best that one can do is to linearize the input-output response provided that some relative degree does exist at a certain point. In this case the state space response cannot be completely linearized resulting in zero dynamics (Byrnes and Isidori, 1984; Isidori, 1989). We are primarily interested in full state space linearization whenever possible.

The MIMO linearization problem requires the system's vector relative degree (sometimes called characteristic numbers) to be satisfied such that $r_{1}+\cdots+r_{m}=n$. Then the standard MIMO lin- 
earizing feedback (see Ref. Isidori (1989) for details) can be chosen to exactly linearize the state space as well as the input-output relationship in appropriately chosen coordinates. In this paper, we're interested in systems of the form (1) that do not satisfy this relative degree condition. It has been shown Isidori (1989) that this vector relative degree condition is necessary and sufficient for exact state space linearization. Our approach, however, is to transform the MIMO system to a SISO system via state feedback at all but one of the input-output pairs. The feedback functions are chosen to satisfy the necessary conditions to achieve the proper relative degree for the SISO linearization problem.

We present two examples of multi-input systems that are not feedback linearizable in a MIMO sense, but with our method they can be linearized in a SISO sense. It should be noted that the idea of feedback to all but one input/output channel to enhance some property (e.g., controllability, observability, etc.) from the standpoint of the remaining input/output channel is well known in decentralized control for linear subsystems (see Ref. Corfmat and Morse (1976)).

\section{DESIGN STEPS}

For discussion purposes, the standard relative degree definition for a SISO system is repeated here Isidori (1989). A SISO system $(m=1)$ has a relative degree $r$ at a point $x^{0}$ if

$$
L_{g} L_{f}^{k} h(x)=0
$$

for all $x$ in a neighborhood of $x^{0}$ and all $k<r-1$ and

$$
L_{g} L_{f}^{r-1} h\left(x^{0}\right) \neq 0 .
$$

The method takes systems of the form (1) for which linearization is desired and transforms them to SISO systems via state feedback. The principle is as follows. Choose feedback functions $u_{i}=K_{i}(x), i=1, \ldots, m-1$ such that the SISO relative degree of the $m$ th output channel is equal to $n$. We formally state this as follows.

Definition 1 The Feedback Linearization Enhancement Problem for (1) is to find feedback functions $u_{i}=K_{i}(x), \Gamma$ is the index set of all such $i, \Gamma \subset\{1, \ldots, m\}$, such that the SISO system

$$
\begin{aligned}
\dot{x} & =f(x)+\sum_{i \in \Gamma} g_{i}(x) K_{i}(x)+g_{j}(x) u_{j} \\
y_{j} & =h_{j}(x)
\end{aligned}
$$

has relative degree $r=n$ about some point $x^{0}$ for at least one $j \notin \Gamma$.
Remark 1 The above problem seeks to linearize the input-state response and the input-output response with respect to the $j$ th input-output pair in a neighborhood of $x^{0}$. Thus, the input-output responses at the other $m-1$ input-output pairs will remain nonlinear in the linearizing coordinates. Presumably one would choose the $j$ th input-output pair to be the channel of most interest to the designer. That is, one may wish for one particular input-output response to be linearized while the others are employing state feedback to enable this linearization. This structure is illustrated in Fig. 1.

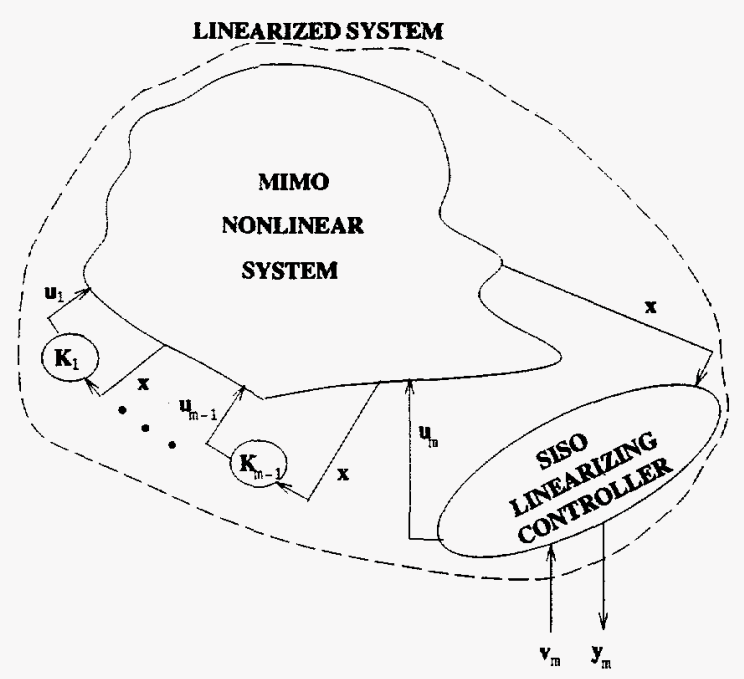

Fig. 1. Illustration of the feedback method for linearization.

The main idea behind the solution of the problem is to choose the feedback functions $K_{i}(x)$ to solve the partial differential equations (2), (3). One normally would only attempt to solve this problem if the MIMO exact linearization problem is not solvable. If the vector relative degree condition for state space linearization is met then one can obtain linearization of all input-output pairs via the MIMO linearizing feedback (see Isidori (1989)). The following theorem states the conditions for solvability of the feedback linearization enhancement problem.

Theorem 1 Necessary conditions for the solvability of the Feedback Linearization Enhancement Problem for (1) $(n>1)$ are:

i.) there must exist at least one $i \in\{1, \ldots, m\}$ such that $L_{g_{i}} h_{i}=0$.

ii.) for at least one of these $i$, there must exist at least one $j \in\{1, \ldots, m\}, j \neq i$ such that $L_{g_{j}} h_{i} \neq 0$.

Proof: Consider the system (1). Without loss of generality, choose $i=m$ as the open input-output channel. We then obtain the equivalent SISO sys- 
tem

$$
\begin{aligned}
& \dot{x}=f(x)+\sum_{j=1}^{m-1} g_{j}(x) K_{j}(x)+g_{m}(x) u_{m} \\
& y_{m}=h_{m}(x) .
\end{aligned}
$$

Unless the system is first order (which is unrealistic for a MIMO system), the relative degree conditions for state space linearization require $L_{g_{m}} h_{m}=0$. This would have to be the case for any $i \in\{1, \ldots, m\}$ one would wish to choose as the open input-output channel. If not the case, then no feedback functions, $K_{i}(x)$, can enhance the relative degree since $r=1$ in this case. This proves condition i.) is necessary.

Assuming condition i.) is true (with $i=m$ ), we continue to the function $L_{\tilde{f}} h_{m}$ where

$$
\begin{aligned}
\tilde{f}(x) & =f(x)+g_{1}(x) K_{1}(x)+\cdots \\
& +g_{m-1}(x) K_{m-1}(x)
\end{aligned}
$$

Again, assuming that the relative degree of the $m$ th input-output channel is insufficient, we require that at least one $K_{i}(x)$ be able to satisfy the relative degree conditions. That is, we require $L_{f} h_{m} \neq 0$. This implies $L_{f} h_{m}+$ $\sum_{j=1}^{m-1}\left(L_{g_{j}} h_{m}\right) K_{j} \neq 0$. From this it can be seen that at least one $j \in\{1, \ldots, m-1\}$ must exist such that $L_{g_{j}} h_{m} \neq 0$ otherwise no $K_{j}$ will be available to enhance the relative degree. This shows that condition ii.) is necessary.

Remark 2 The necessary conditions in the above theorem are easy to check on most systems, however they are not sufficient. The above theorem is meant to eliminate classes of systems for which the method would prove fruitless. The problem of relative degree enhancement is most effectively demonstrated by example.

\section{EXAMPLES}

Example 1 Consider the following third-order nonlinear system

$$
\begin{aligned}
\dot{x} & =\left[\begin{array}{c}
\sin x_{1}+x_{2}^{3} \\
x_{2}^{2}+e^{-x_{3}} \\
x_{1}^{2}+x_{3}
\end{array}\right] \\
+ & {\left[\begin{array}{c}
x_{1} \\
0 \\
1
\end{array}\right] u_{1}+\left[\begin{array}{l}
1 \\
0 \\
0
\end{array}\right] u_{2} }
\end{aligned}
$$

$$
y_{1}=x_{1}, y_{2}=x_{3} \text {. }
$$

A check of this system's relative degree yields $r_{1}=1$ and $r_{2}=1$ which is not sufficient for the MIMO feedback linearization problem to be solvable. However, $L_{g_{2}} h_{2}=0$, thus let $u_{1}=K_{1}(x)$. This results in the new SISO system

$$
\begin{aligned}
\dot{x} & =\left[\begin{array}{c}
\sin x_{1}+x_{2}^{3}+x_{1} K_{1}(x) \\
x_{1}^{2}+e^{-x^{3}} \\
x_{1}^{2}+x_{3}+K_{1}(x)
\end{array}\right] \\
& +\left[\begin{array}{l}
1 \\
0 \\
0
\end{array}\right] u_{2} \\
y_{2} & =x_{3}
\end{aligned}
$$

where $\tilde{g}=\left[\begin{array}{lll}1 & 0 & 0\end{array}\right]^{T}$ and $\tilde{h}=x_{3}$.

Since $L_{\tilde{g}} \tilde{h}=0$, we proceed to the next step of the SISO relative degree definition which requires

$$
L_{\tilde{g}} L_{\tilde{f}} \tilde{h}=2 x_{1}+\frac{\partial K_{1}}{\partial x_{1}}=0 .
$$

This can be satisfied by letting $K_{1}(x)=-x_{1}^{2}+$ $K_{1}^{\prime}\left(x_{2}, x_{3}\right)$. Finally, we require

$$
L_{\tilde{g}} L_{\tilde{f}}^{2} \tilde{h}=2 x_{1} \frac{\partial K_{1}^{\prime}}{\partial x_{2}} \neq 0
$$

which can be solved with (among infinitely many solutions) $K_{1}^{\prime}\left(x_{2}, x_{3}\right)=e^{-x_{3}}$. This results in the feedback function

$$
K_{1}(x)=-x_{1}^{2}+e^{-x_{2}} .
$$

Substituting (15) into (12) does indeed yield $r=$ $n=3$ as required. Furthermore, the point $x^{0}$ about which this result holds is any $\left(x_{1}^{0}, x_{2}^{0}, x_{3}^{0}\right)$ such that $x_{1}^{0} \neq 0$.

Remark 3 The above example shows that the feedback function $K_{i}(x)$ that solves the problem (if one exists) is far from unique. One may have a great deal of freedom to choose feedback functions that are easier to compute or require only readily available states. For instance, in the above example, one could have chosen $K_{1}^{\prime}=x_{2}$ which is less expensive to compute than an exponential in a real-time control situation. However, one would then have to impose $x_{2}^{0} \neq 0$ as an additional requirement on the operating point $x^{0}$.

Example 2 Next, consider the fourth-order system

$$
\dot{x}=\left[\begin{array}{c}
x_{1}^{3}+x_{2} \\
x_{2} x_{3}+x_{4}^{2} \\
x_{1}+x_{2}+x_{1} x_{4} \\
x_{1}^{2}+x_{3} x_{4}
\end{array}\right]+\left[\begin{array}{c}
0 \\
x_{2} \\
1 \\
0
\end{array}\right] u_{1}
$$




$$
+\left[\begin{array}{l}
0 \\
0 \\
0 \\
1
\end{array}\right] u_{2}
$$

$$
y_{1}=x_{1}, y_{2}=x_{3} .
$$

Determination of the system's vector relative degree yields $r_{1}=2$ and $r_{2}=1$, but the input-output decoupling matrix as defined in Ref. Isidori (1989) is singular. Thus, this system has no relative degree, and the state space response cannot be exactly linearized. However, $L_{g_{i}} h_{i}=0$ for $i=1,2$. We choose to let $u_{1}=K_{1}(x)$ which results in the SISO system

$$
\begin{aligned}
& \dot{x}=\left[\begin{array}{c}
x_{1}^{3}+x_{2} \\
x_{2} x_{3}+x_{4}^{2}+x_{2} K_{1}(x) \\
x_{1}+x_{2}+x_{1} x_{4}+K_{1}(x) \\
x_{1}^{2}+x_{3} x_{4}
\end{array}\right] \\
&+ {\left[\begin{array}{l}
0 \\
0 \\
0 \\
1
\end{array}\right] u_{2} } \\
& y_{2}=x_{3}
\end{aligned}
$$

where $\tilde{g}=\left[\begin{array}{llll}0 & 0 & 0 & 1\end{array}\right]^{T}$ and $\tilde{h}=x_{3}$.

Again, we have $L_{\tilde{g}} \tilde{h}=0$. Proceeding as before, we require

$$
L_{\tilde{g}} L_{\tilde{f}} \tilde{h}=x_{1}+\frac{\partial K_{1}}{\partial x_{4}}=0
$$

which has the solution $K_{1}(x)=-x_{1} x_{4}+$ $K_{1}^{\prime}\left(x_{1}, x_{2}, x_{3}\right)$. Continuing the calculations, we get

$$
L_{\tilde{g}} L_{\tilde{f}}^{2} \tilde{h}=\left(1+\frac{\partial K_{1}^{\prime}}{\partial x_{2}}\right)\left(2 x_{4}-x_{1} x_{2}\right)=0
$$

which results in $K_{1}^{\prime}=-x_{2}+K_{1}^{\prime \prime}\left(x_{1}, x_{3}\right)$. Finally, we have the condition

$$
L_{\bar{g}} L_{\tilde{f}}^{3} \tilde{h}=2 x_{4}-x_{1} x_{2} \neq 0
$$

which does not place a condition on $K_{1}(x)$. The simplest feedback then that we can apply is

$$
K_{1}(x)=-x_{1} x_{4}-x_{2} .
$$

This yields $r=n=4$ as we require, and the operating point $x^{0}$ can be any $\left(x_{1}^{0}, \ldots, x_{4}^{0}\right)$ such that

$$
2 x_{4}^{0}-x_{1}^{0} x_{2}^{0} \neq 0 .
$$

Remark 4 In both examples it is important to note that the state space and input-output re- sponses have been linearized for the $u_{2} / y_{2}$ inputoutput pair only. To realize this linearization, one must carry out the coordinates transformation and linearizing feedback for the SISO system $\left(u_{2} / y_{2}\right)$ as detailed in Ref. Isidori (1989). The $u_{1} / y_{1}$ response will remain nonlinear in general.

Remark 5 We assume that each input-output pair has the full state $x$ available for feedback. Thus, this is not strictly a decentralized technique, however the observer result of Schoenwald and Özgüner (1991) could be combined with this result to produce local control laws. This can be done in the following way. Suppose we are interested in SISO state space linearization at the $\ell$ th input-output channel for the system (1) with the conditions of Theorem 1 satisfied. Let $\Gamma$ be the index set of input-output channels utilizing output feedback to allow observer construction at certain channels. Let $\Omega$ be the index set of these certain channels. Then the input-output channels in $\Omega$ will apply full state feedback (from the observed state constructed at these channels) to enhance the relative degree of the $\ell$ th channel. This system is written as

$$
\begin{aligned}
\dot{x} & =f(x)+\sum_{i \in \Gamma} g_{i}(x) K_{i}^{o}\left(y_{i}\right) \\
& +\sum_{j \in \Omega} g_{i}(x) K_{i}^{e}(x)+g_{\ell}(x) u_{\ell} \\
y_{\ell} & =h_{\ell}(x)
\end{aligned}
$$

where $K_{i}^{o}\left(y_{i}\right)$ is the output feedback necessary to allow observer construction at all $j \in \Omega$, and $K_{i}^{e}(x)$ is the state feedback that enhances the relative degree at the $\ell$ th input-output channel. Since the observer construction at all $j \in \Omega$ requires only signals at those channels, the result is completely decentralized.

\section{CONCLUDING REMARKS}

A new method has been presented for linearizing a nonlinear MIMO system by utilizing feedback at all but one of the input-output channels such that the SISO linearization problem is solvable at the remaining input-output channel. The method has been shown to work via example for systems that are not feedback linearizable in a MIMO sense. Thus, the feedback functions at these input-output channels can be viewed as enhancing the solvability of the SISO feedback linearization problem. The examples show that it is not computationally difficult to find these feedback functions for low-order systems, but becomes more difficult as the system order and the number of input-output pairs rise.

Applications for this strategy would include large- 
scale nonlinear systems such as flexible structures undergoing slewing maneuvers, power systems, aircraft, automotive systems, and space structures.

\section{ACKNOWLEDGEMENTS}

The authors wish to acknowledge the support of the AFOSR under Contract F49620-89-C-0046. The first author also gratefully acknowledges the support of the Ohio Aerospace Institute through a doctoral fellowship.

\section{REFERENCES}

Byrnes, C. I. and A. Isidori (1984). A frequency domain philosophy for nonlinear systems. In: Proceedings of the IEEE Conference on Decision and Control. Las Vegas, NV. pp. 15691573.

Cheng, D., A. Isidori, W. Respondek and T. J. Tarn (1988). Exact linearization of nonlinear systems with outputs. Mathematical Systems Theory 21, 63-83.

Corfmat, J. P. and A. S. Morse (1976). Decentralized control of linear multi-variable systems. Automatica 12(2), 169-182.

Isidori, A. (1989). Nonlinear Control Systems. second ed.. Springer-Verlag. Berlin.

Schoenwald, D. and Ü. Özgüner (1991). Some issues in decentralized control of nonlinear systems. In: Proceedings of the IEEE Conference on Decision and Control. Brighton, UK. 